\title{
GROUP ELEMENTS OF PRIME POWER INDEX
}

\author{
BY \\ REINHOLD BAER
}

The index $[G: g]$ of the element $g$ in the [finite] group $G$ is the number of elements conjugate to $g$ in $G$. The significance of elements of prime power index is best recognized once one remembers Wielandt's Theorem that elements whose order and index are powers of the same prime $p$ are contained in a normal subgroup of order a power of $p$ and Burnside's Theorem asserting the absence of elements of prime power index, not 1 , in simple groups. From Burnside's Theorem one deduces easily that a group without proper characteristic subgroups contains an element, not 1 , whose index is a power of a prime if and only if this group is abelian. In this result it suffices to assume the absence of proper fully invariant subgroups, since we can prove [in \$2] the rather surprising result that a [finite] group does not possess proper fully invariant subgroups if and only if it does not possess proper characteristic subgroups.

A deeper insight will be gained if we consider groups which contain "many" elements of prime power index. We show [in \$5] that the elements of order a power of $p$ form a direct factor if, and only if, their indices are powers of $p$ too; and nilpotency is naturally equivalent to the requirement that this property holds for every prime $p$. More difficult is the determination of groups with the property that every element of prime power order has also prime power index [§3]. It follows from Burnside's Theorem that such groups are soluble; and it is clear that a group has this property if it is the direct product of groups of relatively prime orders which are either $p$-groups or else have orders divisible by only two different primes and furthermore have abelian Sylow subgroups. But we are able to show conversely that every group with the property under consideration may be represented in the fashion indicated.

In $\$ 5$ we study the so-called hypercenter. This characteristic subgroup has been defined in various ways: as the terminal member of the ascending central chain or as the smallest normal subgroup modulo which the center is 1. We may add here such further characterizations as the intersection of all the normalizers of all the Sylow subgroups or as the intersection of all the maximal nilpotent subgroups; and the connection with the index problem is obtained by showing that a normal subgroup is part of the hypercenter if, and only if, its elements of order a power of $p$ have also index a power of $p$.

Notation. All the groups under consideration will be finite.

An element [group] is termed primary, if its order is a prime power;

Received by the editors December 14, 1951. 
and if this prime power is in particular a power of the prime number $p$, then we shall refer to $p$-elements and $p$-groups respectively.

$\{J\}$ is the subgroup generated by the subset $J$ of the group $G$.

$A \cap B$ is the intersection of the sets $A$ and $B$.

$[x, y]=x^{-1} y^{-1} x y$, and $[X, Y]$ is the subgroup generated by the commutators $[x, y]$ with $x$ in $X$ and $y$ in $Y$.

$X Y$ is the totality of elements $x y$ with $x$ in $X$ and $y$ in $Y$.

$Z(G)$ is the center of the group $G$.

$Z(J<G)$ is the centralizer of the subset $J$ in the group $G$. This is the totality of elements $z$ in $G$ which commute with every element $j$ in $J$.

$x^{-1} y x=y^{x}$, and $y^{x}$ is the totality of elements $y^{x}$ with $x$ in $X$.

$[G: S]$ is the index of the subgroup $S$ in the group $G$.

The index $[G: g]$ of the element $g$ in the group $G$ is the number of elements in the set $g^{G}$ of elements conjugate to $g$ in $G$. We recall the important equation

$$
[G: g\rfloor=[G: Z(g<G)] \text {. }
$$

1. Indices. For the convenience of the reader we collect in this section a number of properties of indices of subgroups together with their proofs. These more or less well known properties will be basic for our considerations.

Lemмa 1. If $S$ is a subgroup of $G$, and if $N$ is a normal subgroup of $G$, then $[N: N \cap S]$ is a divisor of $[G: S]$.

Proof. It is a consequence of the second isomorphism law that

$$
N S / N \sim S /(N \cap S)
$$

Consequently

$$
\begin{aligned}
{[G: 1] } & =[G: N S][N S: N][N: N \cap S][N \cap S: 1] \\
& =[G: N S][S: N \cap S][N: N \cap S][N \cap S: 1 \text { । } \\
& =[G: N S][N: N \cap S][S: 1]
\end{aligned}
$$

and this implies that

$$
[G: S]=[G: N S][N: N \cap S]
$$

as we claimed.

Lемма 2. If $N$ is a normal subgroup of $G$, and if $g$ belongs to $N$, then $[N: g]$ is a divisor of $[G: g]$.

Proof. We note that $Z(g<N)=N \cap Z(g<G)$; and now Lemma 2 is an immediate consequence of Lemma 1.

LEMмA 3. If $N$ is a normal subgroup of $G$, and if $g$ is an element in $G$, then $[G / N: N g]$ is a divisor of $[G: g]$. 
Proof. It is clear that

$$
N Z(g<G) / N \leqq Z(N g<G / N) .
$$

Consequently we may deduce from the first isomorphism law that

$$
\begin{aligned}
{[G: N Z(g<G)] } & =[(G / N):(N Z(g<G) / N)] \\
& =[(G / N): Z(N g<G / N)][Z(N g<G / N): N Z(g<G) / N] .
\end{aligned}
$$

Hence $[G / N: N g]$ is a divisor of $[G: N Z(g<G)]$; and this index is a divisor of $[G: g]$, since

$$
[G: Z(g<G)]=[G: N Z(g<G)][N Z(g<G): Z(g<G)] .
$$

This proves our contention.

Lemma 4. If the order of the normal subgroup $N$ of $G$ is divisible by the prime number $p$, then $N$ contains an element of order $p$ whose index is prime to $p$.

Proof. It follows from our hypothesis and Sylow's Theorem that the $p$-Sylow subgroups of $N$ are different from 1. If $S$ is some $p$-Sylow subgroup of $N$, then $S$ is contained in some $p$-Sylow subgroup $P$ of $G$. From $S=P \cap N$ we deduce that $S$ is a normal subgroup of $P$. Every normal subgroup, different from 1, of a $p$-group contains a center element of order $p$. Consequently there exists an element $t$ of order $p$ in $S$ which belongs to the center of $P$. Clearly $Z(t<G)$ contains $P$; and this implies that $[G: Z(t<G)]=[G: t]$ is prime to $p$.

The next two lemmas and their proofs are due to $\mathrm{H}$. Wielandt; see also Wielandt [1].

Lemma 5. If the index of the subgroup $S$ in $G$ is a power of the prime $p$, and if $P$ is a p-Sylow subgroup of $G$, then $S \cap P$ is a $p$-Sylow subgroup of $S$ and $G$ $=S P=P S$.

Proof. If $[G: S]=p^{m}$ and if the maximal power of $p$ dividing the order of $S$ is $p^{n}$, then $p^{n+m}$ is the greatest power of $p$ dividing the order of $G$ so that $p^{n+m}$ is the order of $P$. Denote by $p^{k}$ the order of $S \cap P$. It is clear that $p^{k}$ is a divisor of $p^{n}$. One verifies furthermore that every right coset of $P$ modulo $S \cap P$ is contained in one and only one right coset of $G$ modulo $S$ so that $[P: S \cap P] \leqq[G: S]$. Since therefore $[P: S \cap P]$ is a divisor of $p^{m}$ and $[S \cap P: 1]$ is a divisor of $p^{n}$, and since $p^{n+m}=[P: 1]=[P: S \cap P][S \cap P: 1]$, it follows now that

$$
[P: S \cap P]=p^{m}=[G: S], \quad[S \cap P: 1]=p^{n} .
$$

Consequently $S \cap P$ is a $p$-Sylow subgroup of $S$. Furthermore it follows that every right coset of $G$ modulo $S$ contains a right coset of $P$ modulo $S \cap P$. Hence $G=S P$, and $G=P S$ is seen likewise. 
We recall that $\left\{g^{G}\right\}$ is the smallest normal subgroup of $G$ which contains the element $g$.

Lемма 6. $\left\{g^{G}\right\}$ is a p-group if, and only if, $g$ is a p-element and there exists a normal subgroup $N$ of $G$ which contains $g$ such that $[N: g]$ is a power of $p$.

Proof. The necessity of our conditions is an obvious consequence of the fact that $\left\{g^{G}\right\}$ is a normal subgroup of $G$ and that $\left[\left\{g^{G}\right\}: g\right]$ is a divisor of the order of $\left\{g^{G}\right\}$.

Assume conversely that $g$ is a $p$-element, that $g$ is contained in the normal subgroup $N$ of $G$, and that $[N: g]$ is a power of $p$. Then $[N: Z(g<N)]=[N: g]$ is a power of $p$ too. Since the $p$-element $g$ is in $N$, there exists a $p$-Sylow subgroup $P$ of $N$ which contains $g$. It follows from Lemma 5 that $N=Z(g<N) P$. Hence $g^{N}=g^{P}$. Consequently

$$
\left\{g^{N}\right\}=\left\{g^{P}\right\} \leqq P
$$

so that the normal subgroup $\left\{g^{N}\right\}$ of $N$ is a $p$-group. Since every $\left\{g^{N x}\right\}$ $=\left\{g^{N}\right\}^{x}$ is part of $N$, it follows that $\left\{g^{G}\right\}$ is the product of normal subgroups of $N$ which are $p$-groups. Hence $\left\{g^{G}\right\}$ itself is a $p$-group, as we wanted to show.

2. Direct products of isomorphic simple groups. We recall first that $a s u b$ group $S$ is fully invariant in $G$ if $S^{\sigma} \leqq S$ for every endomorphism $\sigma$ of $G$. Clearly every fully invariant subgroup is characteristic and consequently normal, though the converse is not true, as we shall have occasion to point out later on. In order to construct a fully invariant subgroup that will be of importance to us, we need the following concepts. The normal subgroup $M$ of $G$ is of minimal index in $G$ if $M<G$, and if $[G: M]=[G: N]$ whenever the index $[G: N]$ of the normal subgroup $N$ of $G$ is a factor not 1 of $[G: M]$. It is easily seen that every normal subgroup $M$ of minimal index is a maximal normal subgroup of $G$; and this is equivalent to saying that $G / M$ is a simple group $\neq 1$. But the converse is not true; we shall subsequently construct examples of maximal normal subgroups which are not of minimal index. If $N$ is a normal subgroup of $G$, then we denote by $J(G, N)$ the intersection of all the normal subgroups $H$ of $G$ which satisfy: $G / N \sim G / H$. It is clear that $J(G, N)$ is always a characteristic subgroup of $G$,

LeMMA 1. If $N$ is a normal subgroup of minimal index in $G$, then $J(G, N)$ is a fully invariant subgroup of $G$.

Proof. Suppose that $\sigma$ is an endomorphism of $G$. Denote by $H$ some normal subgroup of $G$ which satisfies $G / H \sim G / N$; and denote by $K$ the totality of elements $x$ in $G$ such that $x^{\sigma}$ belongs to $H$. It is clear that this inverse image $K$ of $H$ is also a normal subgroup of $G$. One verifies furthermore that mapping the coset $X$ of $G / K$ upon the coset $H X^{\sigma}$ of $G / H$ is an isomorphism of $G / K$ into $G / H$. Now we distinguish two cases. 
Case 1. $K=G$.

Then $J(G, N)^{\sigma} \leqq G^{\sigma}=K^{\sigma} \leqq H$.

Case 2. $K<G$.

We have pointed out that $G / K$ is isomorphic to a subgroup of $G / H$. Consequently we find that $[G: K] \neq 1$ is a divisor of $[G: H]=[G: N]$. Since $N$ is supposed to be a normal subgroup of minimal index, it follows that $[G: K]=[G: N]=[G: H]$. Since $G / K$ is isomorphic to a subgroup of the group $G / H$ of equal order, we see now that $G / K$ and $G / H$ are isomorphic groups. Consequently $G / K \sim G / H \sim G / N$; and we deduce $J(G, N) \leqq K$ from the definition of $J(G, N)$. This implies

$$
J(G, N)^{\sigma} \leqq K^{\sigma} \leqq H,
$$

since $K$ is the inverse image of $H$.

Thus we have shown in either case that the isomorphy of $G / H$ and $G / N$ implies $J(G, N)^{\sigma} \leqq H$. Hence $J(G, N)^{\sigma} \leqq J(G, N)$ is a consequence of the definition of $J(G, N)$ as the intersection of all the normal subgroups $H$ with $G / H \sim G / N$. Therefore $J(G, N)$ is a fully invariant subgroup of $G$, as we wanted to show.

CoRollary 1. If $G$ is a soluble group, then $J(G, N)$ is a fully invariant subgroup of $G$ for every maximal normal subgroup $N$ of $G$.

This is an immediate consequence of Lemma 1 , since $[G: M]$ is a prime number for every maximal normal subgroup $M$ of the soluble group $G$.

REMARK 1. The indispensability of the solubility hypothesis in Corollary 1 may be seen from the following simple example. Denote by $S$ a non-abelian simple group, by $p$ a prime divisor of the order of $S$, and by $Z$ a cyclic group of order $p$. Form the direct product $G$ of $S$ and $Z$. Then $S$ and $Z$ are the only proper normal subgroups of $G$ and thus they are the only maximal normal subgroups of $G$. We note that $S$ is normal of minimal index, but $Z$ clearly is not. We find furthermore $Z=J(G, Z)$. But $S$ contains a cyclic subgroup of order $p$. Hence there exist endomorphisms $\sigma$ of $G$ which map $Z$ isomorphically into $S$. For these endomorphisms $\sigma$ we have clearly $J(G, Z)^{\sigma}$ $\$ J(G, Z)$ so that $J(G, Z)$ is not fully invariant.

It will be convenient for some of our discussions to denote by $J(G)$ the intersection of all the maximal normal subgroups of $G$. Clearly $J(G)$ is the intersection of all the $J(G, N)$ with maximal normal $N$. Thus $J(G)$ will certainly be fully invariant whenever every $J(G, N)$ with maximal normal $N$ is fully invariant. The converse is not true, as may be seen from the example of Remark 1 where $J(G)=1$, though some of the $J(G, N)$ with maximal normal $N$ are not fully invariant. However it is easy to modify the example of Remark 1 in such a way that $J(G)$ itself ceases to be fully invariant. We just have to substitute for $Z$ a non-abelian $p$-group and select the simple group $S$ so that $Z$ is isomorphic to a subgroup of $S$. Then one verifies easily that $J(G)=J(Z) \neq 1$ 
and that there exists an endomorphism $\sigma$ of $G$ which maps $Z$ isomorphically into $S$.

Proposition 1. The following properties of the group $G$ are equivalent.

(i) $G$ is the direct product of isomorphic simple groups.

(ii) $G$ does not contain proper characteristic subgroups.

(iii) $G$ does not contain proper fully invariant subgroups.

Proof. The equivalence of (i) and (ii) is well known; see, for instance, Zassenhaus [1, p. 77, Satz 2]. It is clear that (ii) implies (iii). Thus assume finally the validity of (iii). There exists certainly a normal subgroup $M$ of minimal index in $G$. It follows from Lemma 1 that $J(G, M)$ is fully invariant. Since $J(G, M) \leqq M<G$, we deduce $J(G, M)=1$ from (iii). Consider now some minimal normal subgroup $L$ of $G$. Because of $J(G, M)=1$ there exists a normal subgroup $H$ of $G$ such that $G / H \sim G / M$ and $L \$ H$. Since $L$ is a minimal normal subgroup of $G$, this implies $L \cap H=1$; and since $H$ is a maximal normal subgroup of $G$, this implies $L H=G$. Hence $G$ is the direct product of $L$ and $H$, and $L, G / H$, and $G / M$ are consequently isomorphic simple groups. Thus we have shown that every minimal normal subgroup of $G$ is a direct factor of $G$, is a simple group, and is isomorphic to the fixed group $G / M$. From these facts one deduces without difficulty that $G$ is the direct product of isomorphic simple groups. Thus (i) is a consequence of (iii), completing the proof.

Proposition 2. The following properties of the group $G \neq 1$ are equivalent.

(i) $G$ is abelian and the direct product of isomorphic simple groups.

(ii) $G$ is abelian and $G^{p}=1$ for some prime $p$.

(iii) No subgroup of $G$ contains a proper fully invariant subgroup.

(iv) $G$ does not contain proper fully invariant subgroups, but $G$ contains elements, not 1 , of prime power index.

Proof. The equivalence of (i) and (ii) is both well known and fairly obvious. If $G$ has property (ii), then each of its subgroups has property (ii). But groups with property (ii) do not contain proper fully invariant subgroups. Hence (iii) is a consequence of (ii).

Assume now the validity of (iii). Since $G \neq 1$, there exists a prime number $p$ which divides the order of $G$. Consider a $p$-Sylow subgroup $S$ of $G$. Then $S \neq 1$ is a $p$-group without proper fully invariant subgroups. Since simple $p$-groups are cyclic of order $p$, it follows from Proposition 1 that $S$ is a direct product of cyclic groups of order $p$. Hence $S$ is abelian and satisfies $S^{p}=1$ [note that it would be very easy to verify this fact without any reference to Proposition 1]. Denote by $T$ the normalizer of $S$ in $G$. Then $S$ is the totality of $p$-elements in $T$. Hence $S \neq 1$ is a fully invariant subgroup of $T$. We apply condition (iii) to see that $S=T$. The abelian group $S$ is therefore contained in the center of its normalizer. Thus we may apply a theorem of Burnside 
which asserts the existence of a normal subgroup $N$ of $G$ such that $G=S N$ and $1=S \cap N$; see, for instance, Zassenhaus [1, p. 133, Satz 4]. Since $S$ is a $p$-Sylow subgroup of $G$, it follows that $N$ is the totality of elements of order prime to $p$ in $G$. Hence $N$ is a fully invariant subgroup of $G$. Since $S \neq 1$, we have $N<G$ and it follows from (iii) that $N=1$. Thus $G=S$ is an abelian group which satisfies $G^{p}=1$. Hence (ii) is a consequence of (iii), and we have shown the equivalence of conditions (i) to (iii).

If the equivalent conditions (i) to (iii) are satisfied by $G$, then it follows from (iii) that $G$ does not contain proper fully invariant subgroups; and it follows from (ii) that every element in $G$ has index 1 . Thus (iv) is certainly a consequence of the equivalent conditions (i) to (iii). Assume conversely the validity of condition (iv). Then we deduce from Proposition 1 that $G$ is the direct product of isomorphic simple groups $S_{1}, \cdots, S_{n}$. If $x \neq 1$ is an element of prime power index in $G$, then $x=x_{1} \cdots x_{n}$ where $x_{i}$ is a uniquely determined element in $S_{i}$. Since $x \neq 1$, at least one of the $x_{i}$ is different from 1 . One verifies without difficulty that

$$
[G: x]=\left[S_{1}: x_{1}\right] \cdots\left[S_{n}: x_{n}\right],
$$

since every element in $S_{i}$ commutes with every element in $S_{j}$ for $j \neq i$. It follows that $\left[S_{i}: x_{i}\right]$ is a power of a prime too. Thus we have shown that at least one [and hence every one] of the simple groups $S_{i}$ contains an element, not 1 , whose index in $S_{i}$ is a prime power. We apply another theorem of Burnside which asserts that a group is not simple if it contains an element of prime power index different from 1 ; see, for instance, Speiser $[1$, p. 190, Satz 165]. Consequently the simple group $S_{i}$ contains a center element different from 1 , since elements of index 1 are necessarily center elements. Hence every $S_{i}$ is abelian. Consequently (i) is satisfied by $G$; and we have completed the proof of the equivalence of the properties (i) to (iv).

REMARK 2. One verifies without difficulty that it is possible to substitute in conditions (iii) and (iv) everywhere for "fully invariant" the word "characteristic."

COROLlaRY 2. If the group $G$ contains an element of prime power index dif ferent from 1, then $G$ contains a proper fully invariant subgroup.

For if $G$ did not contain proper fully invariant subgroups, then condition (iv) of Proposition 2 would be satisfied so that every element in $G$ would have index 1 which contradicts our hypothesis.

REMARK 3. One verifies easily the equivalence of the following two properties of the group $G$.

(a) $G$ contains an element, not 1, of prime power index.

(b) $G$ contains a subgroup of prime power index whose center is not 1 .

It is clear that (b) is a consequence of the following stronger condition.

(c) $G$ contains a nilpotent subgroup of prime power index. 
[Note that $G$ would be a $p$-group if the nilpotent subgroup occurring in (c) were equal to 1.] It is not without interest to note that (c) is a consequence of condition (ii) of Proposition 2, and that therefore the conditions (i) to (iv) of Proposition 2 are equivalent to the following condition.

(v) $G$ does not contain proper fully invariant subgroups, but contains a nilpotent subgroup of prime power index.

REMARK 4. If the group $G$ contains a nilpotent subgroup of prime power index, then the same is true of all the normal subgroups and quotient groups of $G$. Applying the preceding results [Remark 3], an immediate inductive procedure shows that such a group $G$ is soluble. This solubility criterion is due to Szép [1].

3. Prime power order and prime power index. One verifies without much difficulty that a group is soluble if its elements of prime power order have also prime power index; see Lemma (2) below. It is clear that the converse is not true, and thus the determination of this class of groups will not be without interest. This class of groups is completely characterized by the following

TheOREM. Every element of prime power order in the group $G$ has prime power index in $G$ if, and only if, $G$ is the direct product of groups $G_{1}, \cdots, G_{n}$ with the following properties.

(a) The orders of $G_{i}$ and $G_{j}$ are relatively prime for $i \neq j$.

(b) If $G_{i}$ is not of prime power order, then the order of $G_{i}$ is divisible by exactly two different primes and its Sylow subgroups are abelian.

Proof of sufficiency of conditions (a) and (b). If $G$ is the direct product of subgroups $G_{i}$ satisfying conditions (a) and (b), then every primary element belongs to one of the components $G_{i}$. It is clear that $[G: x]=\left[G_{i}: x\right]$ for $x$ in $G_{i}$. If $G_{i}$ is of order a power of $p$, then every element in $G_{i}$ has index a power of $p$. If $G_{i}$ is not primary, then the primary elements $\neq 1$ in $G_{i}$ belong to abelian Sylow subgroups of prime power index; and again we see that the primary elements in $G_{i}$ have prime power index in $G_{i}$.

The proof of the necessity of our conditions is much more complicated and will be effected in a number of steps. It will be convenient to call a group $G$ a $J$-group, if

(J) elements of prime power order in $G$ have prime power index in $G$.

We are now going to derive a number of properties of $J$-groups some of which will be of interest beyond their immediate application.

(1) Normal subgroups and quotient groups of J-groups are J-groups.

Proof. It is a consequence of $\$ 1$, Lemma 2 that normal subgroups of $J$-groups are $J$-groups. If $N$ is a normal subgroup of the $J$-group $G$, then every primary element in $G / N$ has the form $N x$ with $x$ a primary element in $G$; and now it follows from $\S 1$, Lemma 3 that $G / N$ is a $J$-group.

(2) J-groups are soluble.

Proof. It is a consequence of $\S 2$, Proposition 2 that $J$-groups without fully 
invariant subgroups are abelian. It follows from (1) that the principal factors of a $J$-group are $J$-groups without fully invariant subgroups. Hence $J$-groups are soluble.

If $G$ is a group and $p$ a prime, then we may form the product $P=P(p)$ $=P(p, G)$ of all the normal $p$-subgroups of $G$. Clearly $P$ is a normal $p$-subgroup of $G$; and $P$ is the intersection of all $p$-Sylow subgroups of $G$.

(3) $P(p, G)$ contains every element in $G$ whose order and index are powers of $p$.

This is an immediate consequence of $\$ 1$, Lemma 6 .

If $p$ is a prime number, and if every primary element in $G$ has index a power of $p$, then we term $G$ a $J$-p-group. These may be characterized as follows.

Proposition 1. $G$ is a $J$-p-group if, and only if, $G$ is the direct product of a p-group and of an abelian group [of order prime to $p$ ].

Proof. The proof of the sufficiency of our condition is simple enough to be left to the reader. Assume therefore that $G$ is a $J$-p-group. It follows from (3) [and Property $\mathrm{J}-p$ ] that $P(p, G)$ contains every $p$-element, so that $P(p)$ is the $p$-Sylow subgroup of $G$ too. Thus $G / P$ is of order prime to $p$. It follows from $\$ 1$, Lemma 3 that $G / P$ has Property J- $p$. Thus the centralizers of primary elements in $G / P$ have indices which are at the same time powers of $p$ and prime to $p$. Hence primary elements in $G / P$ belong to the center of $G / P$, and this shows that $G / P$ is abelian.

Consider now an element $z$ in the center $Z(P)$ of $P(p)$. Then $P$ is contained in the centralizer of $z$ so that the index of $z$ in $G$ is prime to $p$. Since the index of the $p$-element $z$ is a power of $p$, it follows that $z$ has index 1 ; and we have shown that $Z(P) \leqq Z(G)$. By a fairly obvious induction procedure we see now that $G$ is nilpotent, since $G / P$ is abelian; and now it is fairly obvious that $G$ is the direct product of the $p$-group $P$ and of an abelian group of order prime to $p$. This completes the proof.

(4) If the primary element $g$ in the $J$-group $G$ does not belong to $Z[P(p)<G]$, then $[G: g]$ is a power of $p$, not 1 .

Proof. It follows from our hypothesis that $P(p) \leqq Z(g<G)$. Thus $Z(g<G)$ $\$ G$ so that $[G: g] \neq 1$. Furthermore $Z(g<G)$ does not contain any $p$-Sylow subgroup of $G$ so that $[G: Z(g<G)]$ cannot be prime to $p$. But $[G: g]$ is a power of a prime, since $G$ is a $J$-group. Hence $[G: g]$ is a power of $p$, not 1 , as we wanted to show.

Proposition 2. If $G$ is a $J$-group, and if $P(p, G)$ is not abelian, then $P(p, G)$ is a $p$-Sylow subgroup and a direct factor of $G$.

Proof. We note first that $Z=Z[P(p, G)<G]$ is a normal subgroup of $G$ which does not contain $P=P(p, G)$ and that $Z \cap P$ is the center $Z(P)$ of $P$.

Suppose now that $w$ is a $p$-element, not in $P$. Then it follows from (3) 
that $[G: w]$ is prime to $p$. Consequently $Z(w<G)$ contains a $p$-Sylow subgroup of $G$. But $P$ is part of every $p$-Sylow subgroup of $G$. Hence $P \leqq Z(w<G)$ for every $p$-element $w$, not in $P$; and consequently $w$ belongs to $Z$ whenever $w$ is a $p$-element not in $P$. Assume now by way of contradiction the existence of a $p$-element $c$, not in $P$. Then every element in $P c$ is a $p$-element, not in $P$; and it follows from what we have shown just now that $P c$ is part of $Z$. But then $P$ would be part of $Z$, contradicting the noncommutativity of $P$. Thus $P$ contains every $p$-element so that $P$ is the $p$-Sylow subgroup of $G$.

If $x^{*} \neq 1$ is a primary element in $G^{*}=G / Z$, then there exists a primary element $x$ in $G$ such that $x^{*}=Z x$; and it is clear that $x$ does not belong to $Z$. But then it follows from (4) that $[G: x]$ is a power of $p$; and we deduce from $\S 1$, Lemma 3 that $\left[G^{*}: x^{*}\right]$ is a power of $p$. Thus $G^{*}$ is a $J$-p-group; and it follows from Proposition 1 that $G^{*}$ is the direct product of a $p$-group $P^{*}$ and of an abelian group $A^{*}$ whose order is prime to $p$.

Since $P$ has been shown to be the $p$-Sylow subgroup of $G$, we have $P^{*}$ $=P Z / Z$; and there exists a normal subgroup $A$ of $G$ such that $Z \leqq A$ and $A^{*}=A / Z$.

If $x$ is an element in $P$, but not in $Z$, then we deduce from (4) that $[G: x]$ is a power of $p$; and we have clearly $Z=Z(P<G) \leqq Z(x<G)$. Hence $\left[G^{*}: Z(x<G) / Z\right]=[G: Z(x<G)]=[G: x]$ is a power of $p$. Since the order of the normal subgroup $A^{*}$ of $G^{*}$ is prime to $p$, it follows now that $A^{*} \leqq Z(x<G) / Z$ or $A \leqq Z(x<G)$. Consequently $x$ belongs to $Z(A<G)$ whenevery $x$ is an element in $P$, but not in $Z$.

Consider now an element $z$ in $P \cap Z$. Since $P$ is not abelian, there exists an element $t$ in $P$ which does not belong to $Z$. Then $t$ and $t z$ are elements in $P$ which do not belong to $Z$; and it follows from what we have shown in the preceding paragraph that $t$ and $t z$ belong to $Z(A<G)$. Hence $z$ too belongs to $Z(A<G)$.

Combining the results of the last two paragraphs we see that $P \leqq Z(A<G)$. But then we have $Z \leqq A \leqq Z(P<G)=Z$ or $A=Z$. Thus we have in particular $A^{*}=1, G^{*}=P^{*}$, and $G=P Z$.

Since $P$ is the $p$-Sylow subgroup of $G, P \cap Z$ is the $p$-Sylow subgroup of $Z=Z(P<G)$. It is clear that $P \cap Z$ is part of the center of $Z$; and that the order of $P \cap Z$ is relatively prime to $[Z: P \cap Z]$. Thus we may apply Schur's Theorem; see Zassenhaus [1, p. 125, Satz 25]. Consequently $Z$ is the direct product of $Z \cap P$ and of a subgroup $T$ of order prime to $p$. Clearly $P \cap T=1$ and $G=P Z=P(Z \cap P) T=P T$. Since every element in $P$ commutes with every element in $T \leqq Z=Z(P<G)$, we have shown that $G$ is the direct product of $P$ and $T$; and this completes the proof.

Cf. in this context $\$ 5$, Corollary 2 .

The $J$-group $H$ shall be termed a $J^{*}$-group, if every $P(p, H)$ is abelian.

PROPOSITION 2*. Every $J$-group is the direct product of a nilpotent group and a $J^{*}$-group whose orders are relatively prime. 
This is an immediate consequence of Proposition 2, if we only remember that direct products of $p$-groups are nilpotent.

(5) If $G$ is a $J$-group, and if $P(p, G)$ is abelian, then

(5.a) $G / Z[P(p, G)<G]$ is a primary abelian group of order prime to $p$ and

(5.b) every p-element in $G$ has index prime to $p$.

Proof. We let $P=P(p, G)$ and $Z=Z(P<G)$. Since $P$ is abelian, we have $P \leqq Z(P<G)$.

Consider now a $p$-element $g$ in $G$. If $[G: g]$ is a power of $p$, then $g$ belongs to $P$ and hence to $Z$, as follows from (3). If $[G: g]$ is not a power of $p$, then $[G: g]$ is prime to $p$. Hence $[G: Z(g<G)]$ is prime to $p$ so that $Z(g<G)$ contains a $p$-Sylow subgroup of $G$. This implies $P \leqq Z(g<G)$, since $P$ is part of every $p$-Sylow subgroup of $G$. Consequently $g$ belongs to $Z(P<G)$. Thus we have shown that

(5.c) $Z$ contains every $p$-element in $G$.

It follows from (5.c) that $Z$ contains every $p$-Sylow subgroup of $G$; and this implies that

(5.d) $[G: Z]$ is prime to $p$.

Application of (4) and $\S 1$, Lemma 3 shows that $G / Z$ is a $J$-p-group. But then it follows from (5.d) and Proposition 1 that

(5.e) $G / Z$ is an abelian group.

Let $G^{*}=G / Z$. This abelian group is clearly essentially the same as a group of automorphisms of $P$; and $x^{*}$ is a well determined element in $P$ for every $x$ in $P$ and $g^{*}$ in $G^{*}$. In particular $Z\left(x<G^{*}\right)=Z(x<G) / Z$ is the subgroup of automorphisms in $G^{*}$ which leave invariant the element $x$ in $P$. Assume now by way of contradiction that $G^{*}$ is not a primary group. Then $G^{*}$ is the direct product of a $q$-group $Q^{*}$, an $r$-group $R^{*}$, and a group $S^{*}$ of order prime to $q r$ where $q$ and $r$ are different primes and where $Q^{*}$ and $R^{*}$ are both different from 1 . Since $Q^{*}$ is a group of automorphisms of $P$, there exists an element $x$ in $P$ which is not left invariant by $Q^{*}$. Hence $Q^{*}$ is not part of $Z\left(x<G^{*}\right)$ so that $[G: x]=[G: Z(x<G)]=\left[G^{*}: Z\left(x<G^{*}\right)\right]$ is divisible by $q$. Since $G$ is a $J$-group, it follows that $\left[G^{*}: Z\left(x<G^{*}\right)\right]$ is a power of $q$, not 1 . Since $G^{*}$ is abelian, this implies $R^{*} S^{*} \leqq Z\left(x<G^{*}\right)$; and we have consequently $x^{G}=x^{G^{*}}=x^{Q^{*}}$. Similarly there exists an element $y$ in $P$ which is not left invariant by $R^{*}$; and $\left[G^{*}: Z\left(y<G^{*}\right)\right]$ is a power of $r$, not $1, Q^{*} S^{*} \leqq Z\left(y<G^{*}\right)$, and $y^{G}=y^{R *}$. Consider now the element $x y$ in $P$. It is clear that $S^{*}$ $\leqq Z\left(x y<G^{*}\right)$ and that $[G: x y]=\left[G^{*}: Z\left(x y<G^{*}\right)\right]$ is a prime power. Thus $[G: x y]$ is a power of $q$ or a power of $r$; and we may assume without loss in generality that $\left[G^{*}: Z\left(x y<G^{*}\right)\right]=[G: x y]$ is a power of $q$. Since $G^{*}$ is abelian, this implies that $R^{*} \leqq Z(x y<G)$. But $R^{*} \leqq Z\left(x<G^{*}\right)$. Hence $x$ and $x y$ are both left invariant by $R^{*}$ so that $y$ too is left invariant by $R^{*}$, contradicting our choice of $y$. Our assumption that $G^{*}$ is not primary has led us to a contradiction; and this completes the proof of (5.a). 
Consider now a $p$-element $g$ whose index is a power of $p$. It follows from (3) that $g$ belongs to $P$. Hence $Z=Z(P<G) \leqq Z(x<G)$. Since $[G: Z(x<G)]$ $=[G: x]$ is a power of $p$ and a divisor of $[G: Z(P<G)]$, and since the latter index is prime to $p[$ by (5.a)], we have $[G: x]=1$. Now (5.b) is a consequence of the fact that every $p$-element has prime power index.

Proposition 3. The following properties of the J-group $G$ and the prime $p$ are equivalent.

(i) The p-Sylow subgroups of $G$ are abelian.

(ii) $P(p, G)$ is abelian.

(iii) p-elements in $G$ have index prime to $p$.

Proof. (i) implies (ii), since $P(p, G)$ is part of every $p$-Sylow subgroup of $G$; and it follows from (5) that (ii) implies (iii).

We assume finally the validity of (iii). We denote by $H=H(p, G)$ the subgroup of $G$ which is generated by the p-elements in $G$. Clearly $H$ is a characteristic subgroup of $G$; and it follows from $\$ 1$, Lemma 2 that $H$ has property (iii). Denote by $K=K(p, G)$ the product of all the normal subgroups of $H$ whose order is prime to $p$. Clearly $K$ is a characteristic subgroup of $H$ and $G$ whose order is prime to $p$. Let $H^{*}=H / K$. Then it follows from [(1) and] $\S 1$, Lemma 3 that $H^{*}$ is a $J$-group with property (iii).

It follows from (4) and (iii) that $Z\left[P(p, H)^{*}<H^{*}\right]$ contains every $p$-element. But $\left[H\right.$ and consequently] $H^{*}$ is generated by its $p$-elements. Hence $H^{*}=Z\left[P\left(p, H^{*}\right)<H^{*}\right]$. Consequently $P\left(p, H^{*}\right)$ is part of the center of $H^{*}$; and this implies in particular that $P\left(p, H^{*}\right)$ is abelian.

Assume now by way of contradiction that $P^{*}=P\left(p, H^{*}\right)<H^{*}$. Since $H^{*}$ is a $J$-group, it follows from (2) that $H^{*}$ is soluble. Consequently there exists a normal subgroup $N^{*}$ of $H^{*}$ such that $P^{*}<N^{*}$ and $\left[N^{*}: P^{*}\right]$ is a prime power. If $\left[N^{*}: P^{*}\right]$ were a power of $p$, then $N^{*}$ would be a normal $p$-subgroup of $H^{*}$ so that $N^{*} \leqq P\left(p, H^{*}\right)<N^{*}$, an impossibility. Consequently $\left[N^{*}: P^{*}\right]$ is a prime power prime to $p$. Thus $\left[N^{*}: P^{*}\right]$ and the order of $P^{*}$ are relatively prime; and we may apply Schur's Theorem; see Zassenhaus $[1$, p. 125, Satz $25]$. Since $P^{*}$ is part of the center of $H^{*}$, and hence of the center of $N^{*}$, it follows that $N^{*}$ is the direct product of $P^{*}$ and a subgroup $M^{*}$. But $M^{*}$ is now the totality of elements of order prime to $p$ in the normal subgroup $N^{*}$ of $H^{*}$. Hence $M^{*}$ is a characteristic subgroup of $N^{*}$ and a normal subgroup of $H^{*}$. Consequently $M^{*}=M / K$ where $M$ is a normal subgroup of $H, K<M$, and $[M: K]$ prime to $p$. But then the order of the normal subgroup $M$ of $H$ is prime to $p$; and it follows that $M \leqq K<M$, an impossibility. Our assumption that $P^{*}<H^{*}$ has led us to a contradiction; and we have shown that $H^{*}=P^{*}$ is an abelian $p$-group.

Since $H / K$ is a $p$-group, and since the order of $K$ is prime to $p$, we find that $H / K$ is isomorphic to the $p$-Sylow subgroups of $H$. Since $H / K$ is abelian, 
the $p$-Sylow subgroups of $H$ are abelian; and since the $p$-Sylow subgroups of $H$ and $G$ are the same, we have verified the validity of (i). This completes the proof.

It will be convenient to denote by $F(G)$ the product of all the subgroups $P(p, G)$ of $G$. This characteristic subgroup $F(G)$ of $G$ has been introduced by $\mathrm{H}$. Fitting and $\mathrm{E}$. Wendt. It is the most comprehensive nilpotent normal subgroup of $G$.

Proposition 4. If $G$ is a $J^{*}$-group, then $F(G)$ and $G / F(G)$ are abelian and $F(G)=Z[F(G)<G]$.

Proof. Since $G$ is a $J^{*}$-group, every $P(p, G)$ is abelian and consequently their direct product $F(G)$ is abelian too. This implies that $F=F(G) \leqq Z(F<G)$. Assume by way of contradiction that $F<Z(F<G)$. Since $G$ is a $J^{*}$-group, we deduce from (2) the solubility of $G$. Hence we may deduce from $F<Z(F<G)$ the existence of a normal subgroup $W$ of $G$ such that $F<W$ $\leqq Z(F<G)$ and such that $W / F$ is abelian. But $F$ is part of the center of $Z(F<G)$ and hence part of the center of $W$ so that $W$ is a nilpotent normal subgroup of $G$. Since $F$ is the most comprehensive nilpotent normal subgroup of $G$, we find that $W \leqq F<W$, an impossibility. Thus we have shown that $F(G)=Z[F(G)<G]$.

Every $P(p, G)$ is abelian. Hence it follows from (5.a) that $G / Z[P(p, G)$ $<G]$ is abelian. The commutator subgroup $[G, G]$ of $G$ is consequently part of every $Z[P(p)<G]$. Since $F(G)$ is the product of all the $P(p), Z[F(G)<G]$ $=F(G)$ is the intersection of all the $Z[P(p)<G]$ each of which contains $[G, G]$. Hence $[G, G] \leqq F(G)$ so that $G / F(G)$ is abelian.

(6) If the p-Sylow subgroup $S$ of the $J^{*}$-group $G$ is not part of $F(G)$, then there exists one and only one prime $q \neq p$ with the following properties.

(a) $\operatorname{SP}(q, G)$ is a characteristic subgroup of $G$.

(b) $S \$ Z[P(q)<G]$.

(c) $S \leqq Z[P(r)<G]$ for every prime $r \neq q$.

Proof. We note first that $S F(G) / F(G)$ is the $p$-Sylow subgroup of the abelian group $G / F(G)$ [Proposition 4]. Thus $S F(G)$ is a characteristic subgroup of $G$. Next we deduce from Proposition 4 and our hypothesis that $S \npreceq F(G)=Z[F(G)<G]$. Consequently there exists a primary element in $F(G)$ which does not commute with every element in $S$; and this implies the existence of a prime number $q$ such that $S \$ Z[P(q)<G]$. Since $S$ is abelian [by Proposition 3], and since $P(p)<S$, it is clear that $p \neq q$, and that $S \leqq Z[P(p)<G]$.

Consider now a prime number $r$ which is different from $p$ and $q$. Let $T=S \cap Z[P(q)<G]$. Then it follows from our choice of $q$ that $T<S$. If the element $s$ in $S$ does not belong to $T$, then $P(q) \$ Z(s<G)$. The prime power $[G: s]=[G: Z(s<G)]$ is consequently divisible by $q$ so that $[G: s]$ is a power of $q$, different from 1 . This implies that $Z(s<G)$ contains an $r$-Sylow subgroup of $G$, since the index of $Z(s<G)$ is prime to $r$. But $P(r)$ is part of every 
$r$-Sylow subgroup of $G$. Hence $P(r) \leqq Z(s<G)$. Consequently every element in $S$ which does not belong to $T$ belongs to $Z[P(r)<G]$. Consider now an element $t$ in $T$. Since $T<S$, there exists an element $s$ in $S$ which does not belong to $T$. Then $s$ and $t s$ are elements in $S$ which do not belong to $T$. Hence both $s$ and $t s$ belong to $Z[P(r)<G]$; and this shows that $t$ too belongs to $Z[P(r)<G]$. Thus we have shown that $S \leqq Z[P(r)<G]$. The prime number $q \neq p$ has consequently properties (b) and (c); and it is obvious that $q$ is uniquely determined by these properties.

Denote by $H$ the product of all the $P(r)$ with $r \neq p$ and $r \neq q$. Then $S F(G)=S P(q) H$. We note furthermore that $H$ is a subgroup of the abelian group $F(G)$ and that therefore the order of $H$ is prime to $p q$. The order of $S P(q)$ is of the form $p^{a} q^{b}$. Hence $H \cap S P(q)=1$. The elements in $H$ commute with those in $P(q)$, since $F(G)$ is abelian; and it follows from (c) that the elements in $H$ commute with those in $S$. Thus we see that $S F(G)$ is the direct product of $H$ and $S P(q)$; and this implies that $S P(q)$ is the totality of elements in $S F(G)$ whose order is divisible by $p$ and $q$ only. Consequently $S P(q)$ is a characteristic subgroup of the characteristic subgroup $S F(G)$ of $G$; and this proves (a).

(7) If $S$ is a Sylow subgroup of the $J^{*}$-group $G$, then $[G: Z(S<G)]$ is a prime power.

Proof. Assume that $S$ is a $p$-Sylow subgroup of $G$. If $S=P(p)$, then it follows from (5.a) that $[G: Z(S<G)]$ is a prime power. Assume next that $P(p)<S$. Then $S \$ F(G)$. We deduce from (6) the existence of a uniquely determined prime number $q \neq p$ with the following properties:

$S P(q)$ is a characteristic subgroup of $G$;

$S \leqq Z[P(r)<G]$ if, and only if, $r \neq q$.

Consider now some prime number $h$ different from $p$ and $q$; and denote by $H$ an $h$-Sylow subgroup of $G$. If $H=P(h)$, then $S \leqq Z[P(h)<G]$ so that $H=P(h) \leqq Z(S<G)$. If $P(h)<H$, then we deduce from (6) the existence of a prime number $k \neq h$ with the following properties:

$H P(k)$ is a characteristic subgroup of $G$;

$H \leqq Z[P(r)<G]$ if, and only if, $r \neq k$.

If $q=k$, then neither $S$ nor $H$ would be contained in the normal subgroup $Z[P(q)<G]$ of $G$ so that the index of $Z[P(q)<G]$ would be divisible by the two distinct primes $p$ and $h$. But this is impossible by (5.a). Hence $q \neq k$.

Let $T=S \cap Z[P(q)<G]$. Then $T<S$, since $S \$ Z[P(q)<G]$ by our choice of $q$. If $s$ is an element in $S$, but not in $T$, then $s$ does not commute with every element in $P(q)$. It follows that $P(q) \$ Z(s<G)$ and that therefore $Z(s<G)$ does not contain any $q$-Sylow subgroups of $G$. Hence $[G: Z(s<G)]$ $=[G: s]$ is divisible by $q$; and this implies that $[G: s]=[G: Z(s<G)]$ is a power of $q$, different from 1 . It follows that $Z(s<G)$ contains at least one $h$-Sylow subgroup $H^{\prime}$ of $G$. Since $k \neq q$, it follows that $S \leqq Z[P(k)<G]$. Hence $P(k) \leqq Z(S<G)$; and thus we have shown that $H^{\prime} P(k) \leqq Z(s<G)$. But $H P(k)$ is a characteristic subgroup of $G$; and as such it contains the 
$h$-Sylow subgroup $H^{\prime}$ which is conjugate to $H$. Hence $H P(k)=H^{\prime} P(k)$; and we have shown that $H \leqq Z(s<G)$. Consequently $Z(H<G)$ contains every element $s$ in $S$ which does not belong to $T$.

Consider now an element $t$ in $T$. Since $T<S$, there exists an element $s$ in $S$ which does not belong to $T$. Hence $s$ and $t s$ are elements in $S$ which do not belong to $T$. It follows that $s$ and $t s$ both belong to $Z(H<G)$. Hence $t$ belongs to $Z(H<G)$. Thus we have shown that $S \leqq Z(H<G)$; and this implies $H \leqq Z(S<G)$.

Since $S$ is abelian, we have $S \leqq Z(S<G)$. Combining this remark with the result of the preceding paragraphs we see: If $r$ is a prime number different from $q$, then $Z(S<G)$ contains an $r$-Sylow subgroup. But then $[G: Z(S<G)]$ is a power of $q$, and this completes the proof of (7).

Proof of necessity of conditions (a) and (b) of Theorem. If $G$ is a $J$-group, then it follows from Proposition $2^{*}$ that $G$ is the direct product of a nilpotent group $N$ and a $J^{*}$-group $M$ whose orders are relatively prime. Naturally $N$ is the direct product of its primary components.

Consider now a $p$-Sylow subgroup $S \neq 1$ of the $J^{*}$-group $M$.

Case 1. $S \leqq Z(M)$.

Since the order of $S$ is relatively prime to the index of $S$ in $M$, and since $S$ is part of the center of $M$, we may apply Schur's Theorem; see Zassenhaus $[1$, p. 125 , Satz 25]. It follows that $M$ is the direct product of $S$ and of a subgroup of order prime to $p$.

Case 2. $S \$ Z(M)$.

Since $M$ is a $J^{*}$-group, $S$ is abelian [Proposition 3]. Hence $S \leqq Z(S<M)$ $<M$; and it follows from $(7)$ that $[M: Z(S<M)]$ is a prime power $q^{m} \neq 1$ with $q \neq p$.

Suppose now that $Q$ is a $q$-Sylow subgroup of $M$. Assume by way of contradiction that $Z(Q<M)$ contains a $p$-Sylow subgroup $S^{\prime}$ of $M$. Then $Q$ is part of $Z\left(S^{\prime}<M\right)$. Since any two $p$-Sylow subgroups of $M$ are conjugate in $M$, there exists an element $x$ in $M$ such that $x^{-1} S^{\prime} x=S$. Then $x^{-1} Q x$ $\leqq x^{-1} Z\left(S^{\prime}<M\right) x=Z\left(x^{-1} S^{\prime} x<M\right)=Z(S<M)$. But the index $[M: Z(S<M)]$ is a power of $q$, different from 1 , so that $Z(S<M)$ cannot contain any $q$-Sylow subgroup of $M$. This contradicts the fact that $x^{-1} Q x$ is a $q$-Sylow subgroup of $M$; and thus we have shown that $Z(Q<M)$ does not contain any $p$-Sylow subgroup of $M$. We apply (7) and see that $[M: Z(Q<M)]$ $=p^{n} \neq 1$ for every $q$-Sylow subgroup $Q$ of $M$.

Since $M$ is a $J^{*}$-group, it follows from (2) that $M$ is soluble. Consequently $Z(S<M)$ is soluble. Denote by $j$ the greatest divisor of the order of $M$ which is prime to $p$ and $q$. From $[M: Z(S<M)]=q^{m}$ it follows that $j$ is the greatest divisor of the order of $Z(S<M)$ which is prime to $p$ and $q$. Applying a Theorem of $\mathrm{Ph}$. Hall we see that $Z(S<M)$ contains a subgroup $A$ of order $j$; see, for instance, Zassenhaus [1, p. 127, Aufgabe 1]. Similarly it follows that $Z(Q<M)$ contains a subgroup $B$ of order $j$. 
Since $A$ and $B$ are subgroups of the order $j$ of the soluble group $M$, and since by our choice of $j$ we find that $[M: A]=[M: B]$ is prime to $j$, it follows from a Theorem of $\mathrm{Ph}$. Hall that $A$ and $B$ are conjugate subgroups of $M$; see, for instance, Zassenhaus [1, p. 127, Aufgabe 3]. Consequently there exists an element $y$ in $M$ such that $y^{-1} B y=A$. Then we let $Q^{\prime}=y^{-1} Q y$; and we find that $A=y^{-1} B y \leqq y^{-1} Z(Q<M) y=Z\left(Q^{\prime}<M\right)$.

The Sylow subgroups $S$ and $Q^{\prime}$ are consequently both contained in $Z(A<M)$ so that the order of $Z(A<M)$ is divisible by $o(S) o\left(Q^{\prime}\right)=[M: A]$. We deduce from $\mathrm{Ph}$. Hall's Theorem the existence of a subgroup $D$ of the soluble group $Z(A<M)$ whose order $o(D)=[M: A]$, since $[M: A]$ and $o(A)$ $=j$ are relatively prime. Since every element in $D$ commutes with every element in $A$, and since $o(M)=[M: A][A: 1]=o(D) o(A)$, one verifies without difficulty that $M$ is the direct product of $A$ and $D$. The orders of $A$ and $D$ are relatively prime. Since $D$ contains every $p q$-element of $M$, we have $D=S Q^{\prime}$ and the order of $D$ is divisible by the two primes $p$ and $q$ only. Finally we deduce from Proposition 3 that the Sylow subgroups $S$ and $Q^{\prime}$ of the $J^{*}$-group $M$, and of $D$, are abelian.

Combining the results of Cases 1 and 2 one verifies by an obvious inductive argument-using the fact that by (1) direct factors of $J^{*}$-groups are also $J^{*}$-groups-that $M$ is the direct product of groups of relatively prime orders whose Sylow subgroups are abelian and whose orders are divisible by no more than two prime numbers each. This completes the proof of our theorem.

REMARK. One proves without too much difficulty that a group $G$ is nilpotent if, and only if, every $p$-element in $G$ has index a power of $p$; for instance, by an application of $\$ 1$, Lemma 6 . This criterion is also a special case of our present theorem; and it will appear as a special case of some later results too.

The question may be raised as to the characterization of those groups whose $p$-elements, for just one prime $p$, have prime power index. The present discussion does not seem to throw much light on this question.

4. Hypercenter and hypercommutator. The hypercenter of the group $G$ is the intersection $H(G)$ of all the normal subgroups $N$ of $G$ with the property:

(1) The center of $G / N$ is 1 .

It is not difficult to see that the center of $G / H(G)$ is 1 . If one defines inductively the ascending center chain $Z_{i}(G)$ of $G$ by the rule

(2) $Z_{0}(G)=1, Z_{i+1}(G) / Z_{i}(G)$ is the center of $G / Z_{i}(G)$,

then this ascending chain of normal subgroups of $G$ terminates after a finite number of steps; and it is not difficult to see that

(3) $H(G)=Z_{i}(G)$ for almost every $i$.

It is clear that the hypercenter $H(G)$ is a characteristic subgroup of $G$; and it is not difficult to construct examples showing that $H(G)$ need not be fully invariant. We note finally that $H(G)$ is nilpotent. For the elementary prop- 
erties of the hypercenter see, for instance, Baer [2, p. 20].

The hypercommutator of the group $G$ is the intersection $C(G)$ of all the normal subgroups $N$ of $G$ with the property:

(4) $G / N$ is nilpotent.

It is not difficult to see that $G / C(G)$ itself is nilpotent. If one remembers that a group is nilpotent if, and only if, it is the direct product of its primary components, then one verifies that

(5) $C(G)$ is the intersection of all the normal subgroups with prime power index in $G$.

If we remember next that a group is nilpotent if, and only if, elements of relatively prime order commute, then we see that

(6) $C(G)$ is generated by the commutators $x^{-1} y^{-1} x y$ where $x$ and $y$ are elements of relatively prime order in $G$.

This characterization (6) of $C(G)$ shows that $C(G)$ is a fully invariant subgroup of $G$. If we define the descending center chain ${ }^{i} G$ of $G$ inductively by the rules

(7) ${ }^{0} G=G,{ }^{i+1} G=\left[G,{ }^{i} G\right]$, then this descending chain of normal subgroups of $G$ terminates after a finite number of steps; and it is not difficult to see that

(8) $C(G)={ }^{i} G$ for almost every $i$.

From this last property one deduces finally that

(9) $C(G)$ is the product of all the normal subgroups $N$ of $G$ such that $N$ $=[G, N]$.

For the various characteristic properties of nilpotent groups see Baer [1] and Zassenhaus [1, pp. 104-109, 123]; and for the elementary properties of the hypercommutator see, for instance, Baer [2, p. 20].

$$
[C(G), H(G)]=1 .
$$

Proof. It is well known that

$$
\left[{ }^{i} G, Z_{j}(G)\right] \leqq Z_{j-i-1}(G)
$$

for $i+1 \leqq j$;

see, for instance, Zassenhaus [1, p. 121, (10)]; and now (10) is an immediate consequence of (3) and (8).

Because of this relation (10) we shall be interested in the centralizer $D(G)$ $=Z[C(G)<G]$ of the hypercommutator in $G$. Clearly $H(G) \leqq D(G)$ and $D(G)$ is a characteristic subgroup of $G$.

(11) $D(G)$ is nilpotent.

Proof. Since $G / C$ is nilpotent, the subgroup $D C / C$ is nilpotent too; and it follows from the Isomorphism Theorem that $D /(D \cap C) \sim D C / C$ is a nilpotent group. Since every element in $D$ commutes with every element in $C, D \cap C$ is part of the center of $D$. Hence $D$ is nilpotent modulo its center; and this implies clearly the nilpotency of $D$. 
(12) If the orders of the element $d$ in $D$ and the element $g$ in $G$ are relatively prime, then $d^{-1} g^{-1} d g$ belongs to $C \cap D=Z(C) \cap Z(D)$.

Proof. $C d$ and $C g$ are elements of relatively prime order in the nilpotent group $G / C$. Hence they commute so that the commutator $[d, g]$ belongs to $C$; and $[d, g]$ belongs to $D$, since $d$ is an element in the normal subgroup $D$ of $G$. Thus $[d, g]$ belongs to $C \cap D$. But every element in $C$ commutes with every element in $D=Z(C<G)$. Hence $C \cap D \leqq Z(C) \cap Z(D) \leqq C \cap D$ or $C \cap D$ $=Z(C) \cap Z(D)$.

See also $\$ 5$, Corollary 5 .

(13) The following properties of the normal subgroup $N$ of $G$ are equivalent.

(i) $C(G) \leqq N$.

(ii) If $S$ is a minimal subgroup of $G$ with the property $G=N S$, then $S$ is nilpotent.

(iii) There exists a nilpotent subgroup $S$ of $G$ such that $G=N S$.

Proof. Assume first that $C(G) \leqq N$ and that $S$ is a minimal subgroup of $G$ with the property $G=N S$. Then $G / N$ is nilpotent as a quotient group of the nilpotent group $G / C(G)$. Consider now a $p$-Sylow subgroup $P$ of $S$. Then $N P / N$ is a $p$-Sylow subgroup of the nilpotent group $G / N$. Consequently $N P / N$ is a normal subgroup of $G / N$ so that $N P$ is a normal subgroup of $G$. Hence $N P \cap S$ is a normal subgroup of $S$ and we deduce from $P \leqq S$ and Dedekind's Law that $N P \cap S=P(N \cap S)$. Denote by $T$ the normalizer of $P$ in $S$. Then $P \leqq T \leqq S$. If $s$ is an element in $S$, then $s^{-1} P s$ is a $p$-Sylow subgroup of $N P \cap S$, since $N P \cap S$ is a normal subgroup of $S$ which contains the Sylow subgroup $P$ of $S$. Thus $P$ and $s^{-1} P s$ are $p$-Sylow subgroups of $N P \cap S$ and we deduce from Sylow's Theorem the existence of an element $h$ in $N P \cap S$ such that $s^{-1} P s=h^{-1} P h$. From $N P \cap S=P(N \cap S)$ we deduce the existence of elements $u$ and $v$ in $P$ and $N \cap S$ respectively such that $h=u v$. Then

$$
s^{-1} P s=h^{-1} P h=v^{-1} u^{-1} P u v=v^{-1} P v .
$$

Consequently $s v^{-1}$ belongs to the normalizer $T$ of $P$ in $S$ and $s$ belongs to $T v \leqq T(N \cap S)$. Hence $S \leqq T(N \cap S)$ and consequently

$$
G=N S \leqq N(N \cap S) T=N T \text { or } G=N T .
$$

From the minimality of $S$ we infer now $S=T$. Thus we have shown that every Sylow subgroup of $S$ is a normal subgroup of $S$; and this is equivalent with the nilpotency of $S$. Thus (ii) is a consequence of (i).

That (ii) implies (iii) is an obvious consequence of the existence of minimal subgroups $S$ of $G$ satisfying $G=N S$.

If finally $S$ is a nilpotent subgroup of $G$ such that $G=N S$, then $G / N$ $\sim S /(N \cap S)$. Thus $G / N$ is nilpotent, since it is a homomorphic image of the nilpotent group $S$. Now it follows from the definition of $C(G)$ that $C(G) \leqq N$. Thus (i) is a consequence of (iii), completing the proof.

5. The elements and normal subgroups contained in the hypercenter. 
We begin our discussion with a proof of the following fundamental characterization of the p-elements which belong to the hypercenter.

THEOREM 1. The following properties of the p-element $g$ in the group $G$ are equivalent.

(i) $g$ belongs to the hypercenter $H(G)$ of $G$.

(ii) $g x=x g$ for every $x$ in $G$ whose order is prime to $p$.

(iii) $\left[G: Z\left(g^{G}<G\right)\right]$ is a power of $p$.

(iv) $[G: g]$ is a power of $p$ and $C(G) \leqq Z(g<G)$.

Proof. To prove that (ii) is a consequence of (i) we verify the following proposition by complete induction with respect to $n$.

(n) If the p-element $h$ belongs to $Z_{n}(G)$, then $h x=x h$ for every $x$ in $G$ whose order is prime to $p$.

This assertion is certainly true for $n=0$ and $n=1$. Thus we may assume the validity of $(\mathrm{n})$ for some positive $n$. Consider a p-element $h$ in $Z_{n+1}(G)$ and an element $x$ in $G$ whose order is prime to $p$. Since $Z_{n+1}(G) / Z_{n}(G)$ is the center of $G / Z_{n}(G)$, the element $c=[x, h]$ belongs to $Z_{n}(G)$. Since $Z_{n+1}(G)$ is a nilpotent characteristic subgroup of $G$, the $p$-elements in $Z_{n+1}(G)$ form a characteristic $p$-subgroup of $G$; and so it follows that $c$ is a $p$-element in $Z_{n}(G)$. Applying the inductive hypothesis we find that $x c=c x$. Now

$$
x^{-1} h x=h c^{-1}
$$

and therefore

$$
x^{-k} h x^{k}=h c^{-k}
$$

for every positive $k$.

If in particular $m$ is the order of $x$, then we find that

$$
h=x^{-m} h x^{m}=h c^{-m} \quad \text { or } \quad c^{m}=1 .
$$

But $c$ is a $p$-element and $m$ is prime to $p$. Hence $c=1$ so that $h x=x h$ as we wanted to show. This completes the inductive proof of $(n)$.

That (ii) is a consequence of (i) is now readily inferred from (n) if one only remembers that $H(G)=Z_{t}(G)$ for sufficiently large $t$.

Assume next the validity of (ii). Then every element conjugate to $g$ has the property (ii) too. The normal subgroup $Z\left(g^{G}<G\right)$ contains consequently every element of order prime to $p$. Hence $\left[G: Z\left(g^{G}<G\right)\right]$ is a power of $p$. Thus (iii) is a consequence of (ii).

Assume next the validity of (iii). Since $Z\left(g^{G}<G\right)$ is a normal subgroup of prime power index, $G / Z\left(g^{G}<G\right)$ is a primary group and therefore nilpotent. Hence it follows from the definition of the hypercommutator that $C(G)$ $\leqq Z\left(g^{G}<G\right)$. We have furthermore $Z\left(g^{G}<G\right) \leqq Z(g<G)$. Thus $[G: g]$ $=[G: Z(g<G)]$ is a divisor of $\left[G: Z\left(g^{G}<G\right)\right]$. Since the latter number is a power of $p$, so is the former; and we have shown that (iv) is a consequence of (iii). 
Assume now the validity of (iv). Since $G / C(G)$ is nilpotent, and since $[G / C(G): Z(g<G) / C(G)]=[G: Z(g<G)]=[G: g]$ is a power of $p$, it follows that $Z(g<G) / C(G)$ contains all the elements of order prime to $p$ in $G / C(G)$; and this implies that $Z(g<G)$ contains every element of order prime to $p$ in $G$. Thus (ii) is true. Let now $G^{*}=G / H(G)$ and $y^{*}=H(G) y$ for every $y$ in $G$. Then the $p$-element $g^{*}$ in $G^{*}$ meets clearly requirement (ii) and the same holds for every element conjugate to $g^{*}$. If $N^{*}=\left\{g^{*}\right\}$, then $Z\left(N^{*}<G^{*}\right)$ contains every element of order prime to $p$; and this implies in particular that $\left[G^{*}: x^{*}\right]$ is a power of $p$ for every $x^{*}$ in $N^{*}$. Since $N^{*}$ is generated by $p$-elements, its order is divisible by $p$ unless $N^{*}=1$. But if the order of $N^{*}$ were divisible by $p$, then we would infer from $\$ 1$, Lemma 4 that $N^{*}$ would contain an element $w^{*}$ of order $p$ whose index is prime to $p$. Since every element in $N^{*}$ has index a power of $p$, this would imply that $w^{*}$ would have index 1. Thus $w^{*}$ would be an element, not 1 , in the center of $G^{*}=G / H(G)$, an impossibility $[\$ 4,(1)]$. It follows that $N^{*}=1$. Hence $1=g^{*}=H(G) g$ so that $g$ is in $H(G)$. Thus (i) is a consequence of (iv). This completes the proof.

REMARK 1. It should be noted that it is impossible to substitute for (iii) or (iv) the weaker condition:

$[G: g]$ is a power of $p$.

This may be seen from the following simple construction which may be generalized easily. Denote by $A$ the direct product of $p$ cyclic groups of order $p \neq 2$; and denote by $b(1), \cdots, b(p)$ some basis of $A$. Then every permutation of the elements $b(i)$ is induced by one and only one automorphism of $A$. Consequently we may adjoin to $A$ the group of all permutations of the $b(i)$. We then obtain a group $G$ with the following properties:

$A$ is a normal subgroup of $G$;

every inner automorphism of $G$ induces a permutation of the elements $b(1), \cdots, b(p)$;

every permutation of the elements $b(1), \cdots, b(p)$ is induced by an inner automorphism of $G$.

It is clear then that $[G: b(i)]=p$, since the elements $b(1), \cdots, b(p)$ are just the elements conjugate to $b(i)$. On the other hand $b(i)$ does not belong to the hypercenter of $G$, since it does not commute with every element of order prime to $p$ [Theorem 1, (ii)]; for instance, there exists an element of order 2 in $G$ which induces a permutation of the elements $b(1), \cdots, b(p)$, interchanging $b(i)$ and $b(i \pm 1)$. Note that every element $g \neq 1$ in the $p$-group $\{b(i)\}$ has the property $[G: g]=p$.

One may use Theorem 1 to characterize the $p$-component of the hypercenter. For a convenient enunciation of this characterization we denote by $C(G, p)$ the intersection of all the normal subgroups $N$ of $G$ such that $[G: N]$ is a power of $p$. It is clear that $C(G) \leqq C(G, p)$, that $[G: C(G, p)]$ is a power of $p$, and that $[C(G, p): C(G)]$ is prime to $p$. Since $H(G)$ is nilpotent, the 
totality of $p$-elements in $H(G)$ is a characteristic subgroup of $H(G)$ and of $G$, the soc. $p$-component of $H(G)$.

Corollary 1. The p-component of $H(G)$ is the totality of p-elements in $Z[C(G, p)<G]$.

Proof. If $g$ is a $p$-element in $Z[C(G, p)<G]=D(G, p)$, then every $g^{x}$ is a $p$-element in $D(G, p)$. It follows that $C(G, p) \leqq Z\left(g^{x}<G\right)$ for every $x$ in $G$; and this implies $C(G, p) \leqq Z\left(g^{G}<G\right)$. Consequently $\left[G: Z\left(g^{G}<G\right)\right]$ is a power of $p$. Hence Theorem 1 , (iii) is satisfied by $g$ so that $g$ belongs to $H(G)$. If conversely the p-element $g$ belongs to $H(G)$, then it follows again from Theorem 1, (iii) that $\left[G: Z\left(g^{G}<G\right)\right]$ is a power of $p$. But $Z\left(g^{G}<G\right)$ is a normal subgroup of $G$. Consequently $C(G, p) \leqq Z\left(g^{G}<G\right)$ so that $g$ belongs to $D(G, p)$. Thus the $p$-component of $H(G)$ is exactly the totality of $p$-elements in $D(G, p)$, as we wanted to show.

Theorem 2'. The normal p-subgroup $P$ of $G$ is part of the hypercenter $H(G)$ of $G$ if, and only if, $P$ is a direct factor of every subgroup $S$ of $G$ such that $P<S$ and $[S: P]$ is prime to $p$.

Proof. Assume first that $P \leqq H(G)$ and that $S$ is a subgroup of $G$ with the following properties: $P<S$ and $[S: P]$ is prime to $p$. Then $P$ is a normal subgroup of $S$ such that the order of $P$ and the index of $P$ in $S$ are relatively prime. Hence we may deduce from Schur's Theorem the existence of a complement $C$ of $P$ in $S$; see, for instance, Zassenhaus [1, p. 125, Satz 25 ]. From the isomorphy of $C$ and $S / P$ it follows that the orders of the elements in $C$ are prime to $p$; and now we deduce from Theorem 1 that every element in $C$ commutes with every element in $P \leqq H(G)$. Thus $S$ is the direct product of $P$ and $C$. This proves the necessity of our condition.

Assume next the validity of our condition. Consider an element $g \neq 1$ in $G$ whose order is prime to $p$. Then no power of $g$, except 1 , belongs to the p-group $P$. Since $P$ is normal, it follows now that $S=\{P, g\}=P\{g\}$ and that $S / P \sim\{g\}$. This implies in particular that $P<S$ and $[S: P]$ is prime to $p$. We apply our condition to see that $S$ is the direct product of $P$ and some subgroup $T$. One verifies that $T$ is the totality of elements of order prime to $p$ in $S$, since the orders of $P$ and $T$ are relatively prime. Hence $g$ belongs to $T$ so that $g$ commutes with every element in $P$. Thus we have shown that every element in $P$ commutes with every element in $G$ whose order is prime to $p$. Since the elements in $P$ are $p$-elements, we deduce from Theorem 1 that every element in $P$ belongs to $H(G)$. Hence $P \leqq H(G)$, and this complete the proof.

THEOREM 2". The p-subgroup $S$ of $G$ is a normal subgroup of $G$ and part of the hypercenter $H(G)$ of $G$ if, and only if,

(a) $S$ is a normal subgroup of some $p$-Sylow subgroup of $G$ and

(b) $[G: x]$ is a power of $p$ for every element $x$ in $S$. 
Proof. Assume first that $S$ is a normal subgroup of $G$ and a subgroup of the hypercenter. As every $p$-subgroup is contained in some $p$-Sylow subgroup, $S$ is clearly a normal subgroup of some $p$-Sylow subgroup; and the necessity of (b) may be inferred from Theorem 1, (iv).

Assume now the validity of conditions (a) and (b). Then there exists a $p$-Sylow subgroup $P$ of $G$ which contains $S$ as a normal subgroup. Suppose now that the normal subgroup $N$ of $G$ is part of $S \cap H(G)$. If the subgroup $T$ of $G$ contains $N$, then we let $T^{*}=T / N$, and we let $g^{*}=N g$ for every $g$ in $G$. Then we infer from $N \leqq H(G)$ that $H\left(G^{*}\right)=H(G)^{*}$. It is clear that $S^{*}$ is a normal subgroup of the $p$-Sylow subgroup $P^{*}$ of $G^{*}$; note $N \leqq S \leqq P$. Finally we deduce from $\$ 1$, Lemma 3 that $\left[G^{*}: x^{*}\right]$ is a power of $p$ for every $x^{*}$ in $S^{*}$. Our proof is completed if $S^{*}=1$; and thus we may assume that $S^{*} \neq 1$. Since $S^{*}$ is a normal subgroup, not 1 , of the $p$-group $P^{*}$, a well known result in the theory of $p$-groups shows that $S^{*} \cap Z\left(P^{*}\right) \neq 1$. Consider now an element $z^{*}$ in $S^{*} \cap Z\left(P^{*}\right)$. We have pointed out already that $\left[G^{*}: z^{*}\right]=\left[G^{*}: Z\left(z^{*}<G^{*}\right)\right]$ is a power of $p$. But $P^{*} \leqq Z\left(z^{*}<G^{*}\right)$, since $z^{*}$ belongs to the center of $P^{*}$. Since $P^{*}$ is a $p$-Sylow subgroup of $G^{*}$, it follows that $\left[G^{*}: Z\left(z^{*}<G^{*}\right)\right]$ is prime to $p$. Thus we have $\left[G^{*}: z^{*}\right]=1$, since 1 is the only power of $p$ which is prime to $p$. In others words: $z^{*}$ belongs to the center of $G^{*}$. Thus we have shown that

$$
S^{*} \cap Z\left(P^{*}\right) \leqq Z\left(G^{*}\right) \text {. }
$$

If now $M$ is the uniquely determined subgroup of $G$ which contains $N$ and satisfies $M / N=S^{*} \cap Z\left(P^{*}\right)$, then we infer $N<M$ from $S^{*} \cap Z\left(P^{*}\right) \neq 1$. Since $M^{*}$ is part of the center of $G^{*}$, it is a normal subgroup of $G^{*}$. Hence $M$ is a normal subgroup of $G$. Since $N \leqq H(G)$ and $M^{*} \leqq Z\left(G^{*}\right)$, we conclude finally that $M \leqq H(G)$ too.

Using the result just obtained one proves now by an obvious inductive argument that $S$ itself is a normal subgroup of $G$ and is part of the hypercenter $H(G)$. This completes the proof.

Corollary 2. The following properties of the p-Sylow subgroup $S$ of $G$ are equivalent.

(i) $S \leqq H(G)$.

(ii) $S$ is a direct factor of $G$.

(iii) $[G: x]$ is a power of $p$ for every $x$ in $S$.

Proof. Assume first that the $p$-Sylow subgroup $S$ of $G$ is part of the hypercenter $H(G)$. Clearly $S$ is a Sylow subgroup of $H(G)$. But $H(G)$ is nilpotent, and so $S$ is a characteristic subgroup of $H(G)$ and therefore of $G$. Since order and index of $S$ in $G$ are relatively prime, we may apply Theorem $2^{\prime}$. Hence $S$ is a direct factor of $G$ and we have shown that (ii) is a consequence of (i).

If next $G$ is the direct product of $S$ and $T$, then $T \leqq Z(s<G)$ for every $s$ in $S$; and this implies that $[G: s]$ is a power of $p$, since $[G: T]$ is a power of p. Thus (iii) is a consequence of (ii). 
If finally (iii) is satisfied by $S$, then it is clear that $S$ satisfies conditions (a) and (b) of Theorem 2", since $S$ is itself a Sylow subgroup. It follows from Theorem $2^{\prime \prime}$ that $S \leqq H(G)$. Thus (i) is a consequence of (iii); and this completes the proof.

THEOREM 3. The following properties of the normal subgroup $N$ of the group $G$ are equivalent.

(i) $N \leqq H(G)$.

(ii) $Z(N) \leqq H(G)$ and $N \leqq Z[C(G)<G]$.

(iii) If $x$ is in $N$ and $y$ is in $G$, and if the orders of $x$ and $y$ are relatively prime, then $x y=y x$.

(iv) If $U$ is a nilpotent subgroup of $G$, then $N U$ is nilpotent.

(v) $N$ is part of the normalizer of every Sylow subgroup of $G$.

(vi) If $x$ is a p-element in $N$, then [G:x] is a power of $p$.

(vii) There exists a nilpotent subgroup $V$ of $G$ such that $N \leqq V$ and $G$ $=V Z(N<G)$.

Proof. It is a consequence of $\S 4,(10)$ that $[H(G), C(G)]=1$, and this shows that (ii) is an immediate consequence of (i).

Assume now the validity of (ii). If $x$ is a $p$-element in $N \leqq Z[(C(G)<G]$ $=D(G)$, and if the order of the element $y$ in $G$ is prime to $p$, then we deduce from $\S 4$, (12) that $[x, y]$ belongs to $C(G) \cap D(G)$. Since $N$ is normal and $x$ is in $N$, the commutator $[x, y]$ belongs to $N$ too. Consequently $[x, y]$ is an element in

$$
C(G) \cap D(G) \cap N=C(G) \cap N \leqq Z(N<G) \cap N=Z(N) \leqq H(G) .
$$

Thus $x$ commutes with $[x, y]$ and it follows from $x^{-1} y x=y[x, y]^{-1}$ that

$$
x^{-i} y x^{i}=y[x, y]^{-i} \quad \text { for every positive } i .
$$

But $x$ is a $p$-element so that the order of $x$ has the form $p^{m}$. Hence

$$
y=x^{-p^{m}} y x^{p^{m}}=y[x, y]^{-p^{m}} \text { or }[x, y]^{p^{m}}=1 .
$$

Thus $[x, y]$ is a $p$-element in $Z(N) \leqq H(G)$. It follows from Theorem 1, (ii) that $[x, y]$ commutes with every element of order prime to $p$. Hence in particular $[x, y]$ and $y$ are commuting elements. Consequently it follows from $y^{-1} x y=x[x, y]$ that

$$
y^{-i} x y^{i}=x[x, y]^{i}
$$

for every positive $i$.

If $n$ is the order of $y$, then we have therefore

$$
x=y^{-n} x y^{n}=x[x, y]^{n} \text { or }[x, y]^{n}=1 .
$$

But $[x, y]$ has been shown to be a $p$-element and $n$ is prime to $p$. It follows that $[x, y]=1$ or $x y=y x$. Consider now an element $u$ in $N$ and an element 
$v$ in $G$ such that the orders of $u$ and $v$ are relatively prime. Then $u$ is the product of commuting primary elements $u_{i}$ in $N$ whose orders are divisors of the order of $u$. Thus the orders of $u_{i}$ and $v$ are relatively prime; and it follows from what we have shown just now that $u_{i} v=v u_{i}$. But this implies clearly $u v=v u$. Thus (iii) is a consequence of (ii).

Assume next the validity of (iii). Then elements of relatively prime order in $N$ commute; and it is well known that this implies the nilpotency of $N$. In particular $N$ is the direct product of its primary components $N(p)$; and $N(p)$, as the totality of $p$-elements in $N$, is a characteristic subgroup of $N$ and a normal subgroup of $G$. Consider now some nilpotent subgroup $U$ of $G$. Then $U$ is the direct product of its primary components $U(p)$ and it is clear that $N(p) U(p)$ is a $p$-Sylow subgroup of the subgroup $N U$ of $G$. If $u$ is an element in $U$, then $u$ transforms both $N(p)$ and $U(p)$ into themselves, since $N(p)$ is a normal subgroup of $G$ and $U(p)$ is a normal subgroup of $U$. Consider next a primary element $t$ in $N$. If $t$ is a p-element, then $t$ belongs to $N(p)$ and it is clear that $t$ transforms $N(p) U(p)$ into itself. If $t$ is a $q$-element with $p \neq q$, then it follows from (iii) that $t$ commutes with every $p$-element in $G$. Consequently $t$ commutes with every element in $N(p) U(p)$ and again it is clear that $N(p) U(p)$ is transformed into itself by $t$. Thus $N(p) U(p)$ is transformed into itself by every element in $U$ and by every primary element in $N$; and this implies clearly that $N(p) U(p)$ is a normal subgroup of $N U$. Hence every Sylow subgroup of $N U$ is a normal subgroup of $N U$. Consequently $N U$ is nilpotent and we have shown that (iv) is a consequence of (iii).

If (iv) is satisfied by $N$, and if $S$ is a $p$-Sylow subgroup of $G$, then $N S$ is nilpotent. But $S$ is a $p$-Sylow subgroup of $N S$ too; and it follows from the nilpotency of $N S$ that $S$ is a normal subgroup of $N S$. But then $N$ is part of the normalizer of $S$. Hence (v) is a consequence of (iv).

Assume next the validity of (v). If $x$ is a p-element in $N$ and if $S$ is a $p$-Sylow subgroup of $G$, then $S=x^{-1} S x$. Thus $\{S, x\}$ is a $p$-group, proving that $x$ belongs to $S$. The totality $N(p)$ of $p$-elements in $N$ is therefore contained in the $p$-group $S$. Hence $N(p)$ is a $p$-group [for every $p$ ] so that $N$ is nilpotent. Since $N$ is a normal subgroup of $G, N(p)$ too is a normal subgroup of $G$. Consider again a $p$-element $x$ in $N$ and a primary element $y$ in $G$ whose order is prime to $p$. Then $y$ belongs to some Sylow subgroup $Y$ of $G$. It follows from (v) that $x^{-1} Y x=Y$. Hence $[x, y]$ belongs to $Y$. But $x$ is an element in the normal subgroup $N(p)$ of $G$ so that $[x, y]$ belongs to $N(p)$ too. Thus $[x, y]$ belongs to $Y \cap N(p)=1$, since the orders of the primary groups $N(p)$ and $Y$ are relatively prime. Consequently $[x, y]=1$ or $x y=y x$. It follows that $Z(x<G)$ contains all the primary elements of order prime to $p$; and this shows that $[G: x]=[G: Z(x<G)]$ is a power of $p$. Hence (vi) is a consequence of (v).

Assume now the validity of (vi). If $x$ is a p-element in $N$, then $[G: x]$ is a power of $p$; and it follows from $\S 1$, Lemma 6 that $\left\{x^{G}\right\}$ is a $p$-group. Thus 
every $p$-element in $N$ belongs to some normal $p$-subgroup of $N$; and this implies clearly that the totality $N(p)$ of $p$-elements in $N$ is a $p$-subgroup of $N$. As a characteristic subgroup of the normal subgroup $N$ the subgroup $N(p)$ is normal in $G$. Consequently $N(p)$ is a normal subgroup of every $p$-Sylow subgroup of $G$. Thus conditions (a) and (b) of Theorem 2" are satisfied by $N(p)$ so that $N(p) \leqq H(G)$. Since therefore all the primary elements in $N$ belong to $H(G)$ we find that $N \leqq H(G)$. Thus (i) is a consequence of (vi) and we have completed the proof of the equivalence of the first six conditions.

Assume now the validity of the equivalent conditions (i) to (vi). Then $N \leqq Z[C(G)<G]$ so that $C(G) \leqq Z(N<G)$. Consequently $G / Z(N<G)$ is nilpotent and we deduce from $\$ 4,(13)$ the existence of a nilpotent subgroup $U$ of $G$ such that $G=U Z(N<G)$. It follows from (iv) that $V=N U$ is nilpotent. We have clearly $N \leqq V$ and $G=U Z(N<G)=V Z(N<G)$. Thus (vii) is a consequence of (i) to (vi).

Assume finally the validity of (vii). If $x$ is a p-element in $N$, then we have certainly $Z(N<G) \leqq Z(x<G)$. Denote by $V$ some nilpotent subgroup of $G$ such that $N \leqq V$ and $G=V Z(N<G)$. Then $x$ is an element in the nilpotent group $V$ and commutes with every element in $V$ whose order is prime to $p$. If $V^{*}(p)$ is the totality of elements of order prime to $p$ in $V$, then we have shown now that $V^{*}(p) Z(N<G) \leqq Z(x<G)$. From $G=V Z(N<G)$ it follows that $V^{*}(p) Z(N<G) / Z(N<G)$ is the totality of elements of order prime to $p$ in $G / Z(N<G)=V Z(N<G) / Z(N<G)$. Hence $[G: x]=[G: Z(x<G)]$ is a power of $p$; and we have shown that (vi) is a consequence of (vii). This completes the proof of our theorem.

COROLLARY 3. The hypercenter is the intersection of the normalizers of all the Sylow subgroups.

This is an immediate consequence of Theorem 3, (v).

COROLlaRy 4. The hypercenter is the intersection of all the maximal nilpotent subgroups.

Proof. If $M$ is a maximal nilpotent subgroup of $G$, then $M H(G)$ is nilpotent by Theorem 3, (iv) and $M=M H(G)$ is a consequence of the maximality of $M$. Hence $H(G) \leqq M$ so that $H(G)$ is part of the intersection $J$ of all the maximal nilpotent subgroups of $G$. It is clear that $J$ is a characteristic subgroup of $G$. If $U$ is a nilpotent subgroup of $G$, then $U$ is part of some maximal nilpotent subgroup $W$ of $G$. Clearly $J \leqq W$. Hence $J U \leqq W$ and $J U$ is nilpotent as a subgroup of a nilpotent group. Thus Theorem 3, (iv) is satisfied by the normal subgroup $J$ so that $J \leqq H(G) \leqq J$ or $J=H(G)$ as we wanted to show.

CoRollary 5. If the normal subgroup $N$ of $G$ is contained in $Z[C(G)<G]$, then $[N, N] \leqq H(G)$. 
Proof. It follows from $\S 4$, (11) that $Z[C(G)<G]$ is nilpotent. The subgroup $N$ of $Z[C(G)<G]$ is therefore nilpotent too. Hence $N$ is the direct product of its primary components which are normal subgroups of $G$ and $[N, N]$ is the direct product of the commutator subgroups of these primary components of $N$. Consequently we may assume without loss in generality that

$N$ is a $p$-group.

Consider now an element $g$ in $G$ whose order is prime to $p$. If $x$ is an element in $N$, then $x$ is a $p$-element and it follows from $\$ 4,(12)$ that $[x, g]$ belongs to the center $Z(N)$ of $N$. If $x$ and $y$ are any two elements in $N$, then $[x, g],[y, g]$, and $[x y, g]$ belong to $Z(N)$ and satisfy therefore

$$
\begin{aligned}
{[x y, g] } & =y^{-1} x^{-1} g^{-1} x y g=y^{-1}[x, g] g^{-1} y g=[x, g] y^{-1} g^{-1} y g \\
& =[x, g][y, g] .
\end{aligned}
$$

Mapping $x$ in $N$ upon $[x, g]$ in $Z(N)$ [for any fixed $g$ ] constitutes therefore a homomorphism of $N$ into the abelian group $Z(N)$. But such a homomorphism maps $[N, N]$ upon 1 ; and thus we have found that $[c, g]=1$ for every $c$ in $[N, N]$ and every $g$ of order prime to $p$. Thus condition (iii) of Theorem 3 is satisfied by $[N, N]$. Hence $[N, N] \leqq H(G)$, as we wanted to show.

REMARK 2. If we let in Theorem 3 in particular $N=G$, then we obtain some more or less well known criteria for the nilpotency of $G$. Conditions (ii), (iv), and (vii) do not give interesting results; but using the remainder of Theorem 3 we obtain the following criterion.

The following properties of the group $G$ are equivalent.

(a) $G$ is nilpotent.

(b) Elements of relatively prime order in $G$ commute.

(c) Sylow subgroups are normal subgroups.

(d) p-elements have p-power index.

Properties (b) and (c) are, of course, well known; actually property (c) is often used as definition of nilpotency; and property (d) is also a consequence of Corollary 2.

THEOREM 4. The following properties of the minimal normal subgroup $M$ of $G$ are equivalent.

(i) $M \leqq Z(G)$.

(ii) $M \leqq H(G)$.

(iii) $G / M Z(M<G)$ is soluble; $M$ contains an element $t \neq 1$ of prime power index $[G: t]$; and to every prime $p$ there exists an element $x \neq 1$ in $M$ whose index $[G: x]$ is prime to $p$.

(iv) $G / Z(M<G)$ is soluble and to every prime $p$ there exists an element $x \neq 1$ in $M$ whose index $[G: x]$ is prime to $p$. 
Proof. It is clear that (i) implies (ii). If $M \leqq H(G)$, and if $p$ is a prime divisor of the order of $M$, then we infer from $\$ 1$, Lemma 4 the existence of an element $x$ of order $p$ in $M$ whose index $[G: x]$ is prime to $p$. But $p$-elements in the hypercenter have index a power of $p$ [by Theorem 1]. Hence $[G: x]=1$ so that $x$ belongs to the center of $G$. But then it follows from the minimality of $M$ that $M \leqq Z(G)$; and thus we have shown the equivalence of (i) and (ii).

It is obvious that (iii) is a consequence of the equivalent conditions (i) and (ii). Assume now the validity of (iii). As a minimal normal subgroup, $M$ is free of proper characteristic subgroups. But $M$ contains an element $t \neq 1$ such that $[G: t]$ is a prime power. We infer from $\S 1$, Lemma 2 that $[M: t]$ is a prime power too. Thus $\$ 2$, Proposition 2 , (iv) is satisfied by $M$. Hence $M$ is commutative. Consequently $M \leqq Z(M<G)$ or $Z(M<G)$ $=M Z(M<G)$; and now it is clear that (iv) is a consequence of (iii).

Assume now the validity of (iv). Because of the minimality of $M$ we have $M \cap Z(M<G)=1$ or $M$. If $M \cap Z(M<G)=1$, then $M$ would be isomorphic to a subgroup of the soluble group $G / Z(M<G)$. Thus $M$ would be a soluble group without proper characteristic subgroup. But such a group is clearly abelian; and this implies $M \leqq Z(M<G)$, a contradiction. Hence $M \cap Z(M<G)=M$ or $M \leqq Z(M<G)$ so that $M$ is abelian.

Assume next by way of contradiction that $Z(M<G) \neq G$. Then we deduce from the solubility of $G / Z(M<G)$ the existence of a normal subgroup $N$ of $G$ with the following properties:

$$
Z(M<G)<N \text { and } N / Z(M<G) \text { is a } p \text {-group. }
$$

Since $N$ is a normal subgroup of $G$, so is $Z(N<G)$. Hence it follows from the minimality of $M$ that $M \cap Z(N<G)=1$ or $M$. In the latter case we would have $M \leqq Z(N<G)$ so that $N \leqq Z(M<G)<N$, an impossibility. Consequently

$$
M \cap Z(N<G)=1 .
$$

It follows from (iv) that there exists an element $u \neq 1$ in $M$ whose index $[G: u]$ is prime to $p$. From $M \leqq Z(M<G)<N$ and $\S 1$, Lemma 2 we deduce that $[N: u]$ is prime to $p$. But clearly

$$
Z(M<G)=N \cap Z(M<G)=Z(M<N) \leqq Z(u<N) \leqq N .
$$

Thus the number $[N: u]=[N: Z(u<N)]$ which is prime to $p$ is a divisor of the number $[N: Z(M<G)]$ which is a power of $p$. It follows that $[N: u]=1$ so that $u$ belongs to the center of $N$. Thus $Z(N) \cap M \neq 1$, contradicting $M \cap Z(N<G)=1$. Consequently $Z(M<G)=G$ so that $M \leqq Z(G)$. Hence (i) is a consequence of (iii). This completes the proof.

Corollary 6. The socle of $G$ is part of $Z[H(G)<G]$.

Here we define, following Remak, the socle as the product of all the 
minimal normal subgroups. That every minimal normal subgroup $M$ of $G$ is part of the centralizer of the hypercenter is easily seen. For if $M$ is part of the hypercenter, then it follows from Theorem 4 that $M$ is part of the center; and if $M$ is not part of the hypercenter, then $M \cap H(G)=1$ and it is evident again that $M \leqq Z[H(G)<G]$.

\section{BIBLIOGRAPHY}

R. BAER

1. Nilpotent groups and their generalizations, Trans. Amer. Math. Soc. vol. 47 (1940) pp. 393-434.

2. Groups with descending chain condition for normal subgroups, Duke Math. J. vol. 16 (1949) pp. 1-22.

W. BuRNSIDE

1. Theory of groups of finite order, 2d ed., Cambridge, 1911.

A. SPEISER

1. Theorie der Gruppen von endlicher Ordnung, 3d ed., Berlin, 1937.

H. WIELANDT

1. Über des Produkt paarweise vertauschbarer nilpotenter Gruppen, Math. Zeit. vol. 55 (1951) pp. 1-7.

H. ZASSENhaUS

1. Lehrbuch der Gruppentheorie I, Hamburger Math. Einzelschriften vol. 21 (1937).

UNIVERSITY OF ILLINOIS,

URBANA, ILL. 